\title{
Discovering Spatiotemporal Event Sequences
}

\author{
Berkay Aydin, Rafal Angryk \\ Department of Computer Science \\ Georgia State University, Atlanta, GA 30303, USA \\ \{baydin2, rangryk\}@cs.gsu.edu
}

\begin{abstract}
Spatiotemporal event sequences represent the sequences of event types whose spatiotemporal instances frequently follow each other in spatiotemporal context. In this work, we present spatiotemporal event sequence mining from spatiotemporal event datasets that contains evolving region trajectories. We propose two algorithms for discovering spatiotemporal event sequences. We formally define a flexible spatiotemporal follow relationship, introduce various data models for capturing the sequence forming behavior. Lastly, we present an extended experimental evaluation that demonstrates the computational efficiency of our algorithms.
\end{abstract}

\section{Categories and Subject Descriptors}

H.4 [Information Systems Applications]: Spatial- Temporal Systems - Spatial Data Mining and Knowledge Discovery

\section{INTRODUCTION}

In traditional itemset mining, frequent sequence (or sequential pattern) mining refers to discovering a set of attributes persistently appearing over time among the large number of objects [27]. A major category of sequences are event sequences, which represents the implicit relations among the categories of objects [8]. Classical event sequence mining can be useful for understanding the user behavior (by mining sequences from weblogs or system traces) [21], shopping routines of customers (by mining transaction sequences) [24], or the efficiency of business processes (by mining time-ordered managerial and operational activities) [29].

Spatiotemporal event sequences (STES) are the ordered lists (with repetitions) of event types whose instances frequently demonstrate sequence generating behavior. The sequence generating behavior is characterized by the spatiotemporal follow relationship among instances, and it refers to temporal follow relationship with spatial proximity constraints. We are interested in STES mining from event instances with continuously evolving polygon-based represen-

Permission to make digital or hard copies of all or part of this work for personal or classroom use is granted without fee provided that copies are not made or distributed for profit or commercial advantage and that copies bear this notice and the full citation on the first page. Copyrights for components of this work owned by others than ACM must be honored. Abstracting with credit is permitted. To copy otherwise, or republish, to post on servers or to redistribute to lists, requires prior specific permission and/or a fee. Request permissions from permissions@acm.org.

MobiGIS'16, October 31-November 03 2016, Burlingame, CA, USA

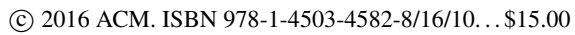

DOI: http://dx.doi.org/10.1145/3004725.3004735 tations. We will introduce two algorithms for the discovery of spatiotemporal event sequences. Within the scope of this paper, we use the term, evolving region trajectories, for moving spatiotemporal objects, whose spatial extensions are represented by evolving polygon-based geometries.

The discovery of STESs is critically important for the verification and prediction of scientific phenomena in a broad range of scientific fields including meteorology, geophysics, epidemiology, and astronomy [9]. The scientific phenomena such as tornadoes, propagation of epidemics, clouds, or solar events can be modeled as evolving region trajectories. STES mining can be used for modeling the spatial and temporal relationships among different types of phenomena. Later, the discovered sequence patterns can be utilized for performing large-scale verification of current knowledge, as well as the prediction of unknown spatiotemporal relationships among different event types. One example application area for STES mining is the space weather prediction. In Fig. 1, we show an active region followed by a sigmoid. Identifying STESs appearing on the sun can help us better understand the relationships among solar event types, and lead to better modeling and forecasting of important events such as coronal mass ejections and solar flares. These events can impact radiation in space, reduce the safety of space and air travel, disrupt intercontinental communication and GPS, and even damage power grids [14].

The task of mining STESs is challenging mainly due to the difficulty of identifying sequence forming instances. From a theoretical standpoint, firstly, a consistent definition for spatiotemporal follow relationship is vital for the accuracy and relevancy of the mining algorithms. Secondly, a meaningful significance measurement for the spatiotemporal follow relationship is necessary. On the other hand, practically, the computational operations required for identifying the sequence forming instances are expensive due to the nested joins with complex spatial and temporal predicates.

Our contributions are as follows: We present preliminary concepts and mining algorithms for the discovery of the STESs. We introduce new models for capturing the sequence forming behaviors among the evolving region trajectories and define a flexible follow relationship. The following issues are beyong the scope of this paper: (1) Determination and statistical analysis of the threshold values for capturing the strength of sequences, (2) Temporal or spatiotemporal indexing techniques related to mining spaiotemporal event sequences, (3) Spatiotemporal event sequence mining from point-based moving objects.

The rest of this paper is organized as follows. In Section 

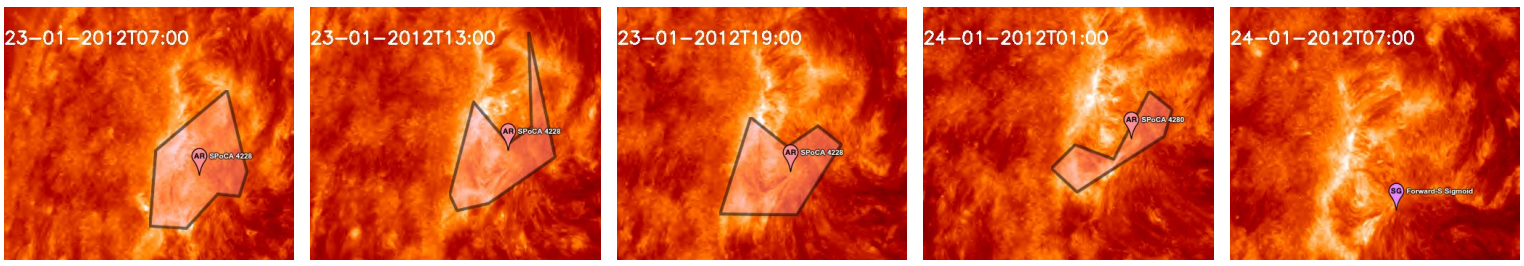

Figure 1: An instance of active region followed by an instance of sigmoid, occurred between 23-01-2012T07:00 to 24-012012T07:00. (The images are obtained from Helioviewer.org, the time and location of the events are overlaid on the images.)

2, we present related work on temporal and spatiotemporal sequence mining. In Section 3, we present definitions for the preliminary concepts, which are basic structures, operations, and measures, used in the mining process. Next, we present our algorithms in Section 4. In Section 5, we present our experimental evaluation. Lastly, in Section 6, we point to possible future work, and conclude the paper.

\section{RELATED WORK}

Over the past two decades, many sequential pattern mining algorithms have been introduced. A comprehensive list of sequential pattern mining algorithms and approaches can be found in a recent survey [15]. Sequential pattern mining algorithms can be investigated under two categories: time point patterns and time interval patterns [16]. In time point patterns, the temporal instances are associated with a specific point in time, while for time interval patterns, they are associated with the interval between two time points. Wellknown sequential pattern mining algorithms for time points include AprioriAll [2], SPADE [27], and PrefixSpan [19].

Related work on time interval patterns can be summarized as follows. Papapetrou et al. used an enumeration tree to discover arrangements (sequences) of interval-based events using a hybrid depth-first and breadth-first search based (HDFS) method [18]. Winarko and Roddick introduced ARMADA, which is a projection-based efficient time interval pattern mining algorithm that utilizes an iterative candidate generation and pruning approach [26]. Wu and Chen proposed TPrefixSpan, which is a modified version of the PrefixSpan algorithm [20] for mining temporal patterns from time interval events. Patel et al. introduced the IEMiner algorithm, which extends H-DFDS method [18] by extending the sequences during the discovery process. Moskovitch and Sharar proposed KarmeLego for the discovery of frequent symbolic time intervals related patterns [17]. KarmaLego uses a temporal abstraction process from raw timestamped data, and utilizes a data structure and exploits the transitivity of Allen's temporal interval algebra [3] for efficient candidate sequence generation.

In the spatiotemporal frequent pattern mining literature, the term sequence (or its derivatives such as sequence patterns, sequential patterns) is used for identifying different types of knowledge from spatiotemporal data. These include sequences of locations visited frequently by spatiotemporal objects [7], sequences of event types whose instances follows each other [12], and sequences of spatiotemporal association rules [25].

Cao et al. describes the spatiotemporal sequential patterns as 'the routes frequently followed by objects' in [7]. Discovered patterns are the frequently visited locations a dataset of spatiotemporal trajectory segments. This work is related to the movement patterns of spatiotemporal objects in the form of trajectory segments. Similarly, Giannotti et al. introduces an algorithm for mining trajectory patterns, which represent a set of trajectories frequently visiting similar locations with similar visiting times [10]. While Giannotti et al.'s work is more focused on the behavioral aspect of spatiotemporal objects, the term, sequence, refers to visited locations. Verhein introduces complex spatiotemporal sequence pattern mining [25] that focuses on sequences of spatiotemporal association rules, which represents frequently occurring movements of spatiotemporal objects appearing between two regions during a specific time interval. This work is interested in discovering the sequences of spatiotemporal meta-rules (movement patterns) for groups of objects.

In [28], Zhang et al. proposed Splitter, which discovers fine-grained sequential patterns from semantic trajectories. The algorithm firstly retrieves spatially coarse patterns, and later reduces them to fine-grained patterns. The discovered is a sequence of categorized locations (deduced from semantic trajectories). Another example of spatiotemporal sequences, called spatio-sequences, are presented by Salas et al. in [23]. The spatio-sequence mining discovers temporal sequences of ordered spatial itemsets that are used for coupling geographically neighboring phenomena.

Huang et al. presented a framework for mining sequential patterns from spatiotemporal event datasets in [12]. The sequential patterns, in [12], refers to a sequence of event types from point-based objects with event type annotations. They formally define a follow relationship between the pointbased instances of different event types, present significance measures for sequences, and introduce two iterative pattern growth algorithms for the mining task. Their algorithms create a pattern tree and expands its nodes with recursively calling tree expansion procedures (i.e., follow joins). It should be noted that sequential pattern mining in [12] considers a totally ordered set of event instances.

Similar to [12], we modeled the sequences of event types from region-based instances in our recent works [5,6]. Our algorithms are not applicable for discovering sequences of locations or movement behaviors. We use the term spatiotemporal event sequence to avoid confusion with other types of existing sequence or sequential pattern definitions.

\section{PRELIMINARY CONCEPTS}

Spatiotemporal event instances are the chronologically ordered lists of timestamp-geometry pairs. The geometries are region-based, and represented with polygons. The instances are essentially evolving region trajectories. They are primarily identified by a unique identifier. Each instance has an associated event type. An event type signifies the class of its associated instances. Formally, an instance is de- 
noted by $i n s_{i}$, time-geometry pairs are denoted by $t g$. A timestamp-geometry pair is a pair of timestamp value $\left(t_{i}\right)$, and a polygon-based region geometry $\left(g_{i}\right)$. The event type of an instance is represented with $i n s_{i} . \mathcal{E}$.

$$
\text { ins }_{i}=\left\{t g_{i_{1}}, t g_{i_{2}}, \ldots t g_{i_{k}}\right\} \text { where } t g_{i}=\left\langle t_{i}, g_{i}\right\rangle
$$

The set of all instances is denoted by $\mathbb{I}=\left\{i n s_{1}, \ldots, i n s_{n}\right\}$. An event type is denoted by $e_{j}$. The set of instances of type $e_{j}$ is represented as $\mathbb{I}_{e_{j}}$

A spatiotemporal event sequence (denoted as $E S$ ) is an ordered series of event types with possible repetitions.

$$
E S_{i}=\left(e_{i_{1}} \triangleright e_{i_{2}} \triangleright \ldots \triangleright e_{i_{k}}\right)
$$

The follow relationship between two event types is denoted by the ' $\triangleright$ ' symbol. This is to say, ' $e_{i} \triangleright e_{j}$ ' indicates $e_{i}$ is followed-by $e_{j}$. Event sequences are derived from instance sequences. An instance sequence (denoted as $I S q$ ) is a unique occurrence of a spatiotemporal event sequence. Instance sequences are formed by individual instances, which follow each other in spatiotemporal context.

$$
I S q_{i}=\left(i n s_{i_{1}}-i n s_{i_{2}} \triangleright \ldots i n s_{i_{k}}\right)
$$

The number of participating instances in an instance sequence is the length of the instance sequence. To refer to the length- $k$ instance sequences, we will use the term $k$-sequence. Given an event sequence $E S_{i}$, an instance sequence $\left(I S q_{i}\right)$ is of-type $E S_{i}$, if and only if the event types of the participating instances of $I S q$ are identical and in the same order as the event types in $E S_{i}$. Namely, following the notation in Eq. 2 and Eq. 3, ins $s_{i_{1}} \cdot \mathcal{E}=e_{i_{1}}, \ldots$, and $i n s_{i_{k}} \cdot \mathcal{E}=e_{i_{k}}$.

\subsection{Head and Tail Window of an Instance}

The instance sequences are formed by two or more instances. Between each two consecutive instances there exists a spatiotemporal follow relationship. Essentially, the follow relationship occurs between two event instances, and is denoted with the ' symbol. The relationship is characterized by two predicates that are temporal continuity and spatial proximity. To actualize these predicates, we present two concepts that are the head and the tail window of instances. The head of an instance refers to the initial segment of the instance's evolving region trajectory. Similarly, the tail of an instance refers to the last segment of the trajectory. Tail window is a complex spatiotemporal buffer obtained by spatially buffering and temporally propagating the tail of an instance. Given an instance, ins $_{i}$, the head and tail window of $i n s_{i}$ are represented with $h_{i}$ and $t w_{i}$, respectively.

Before tail window generation procedure, we will discuss different strategies for head and tail generation. We propose various head and tail generation strategies.

\subsubsection{Interval-based vs. Ratio-based Generation}

In the interval-based generation strategy, we are given two global parameters that are head interval $(h I n)$ and tail interval $(t I n)$. The head interval refers to the time period for determining the head segment of the instance's trajectory. Similarly, the tail interval determines the tail segment of the trajectory. The length of these intervals are fixed for all the instances in a given dataset. This is to say, all the head segments have the same interval length (which is $h I n$ ), and all the tail segments have the same interval length (which is tIn).

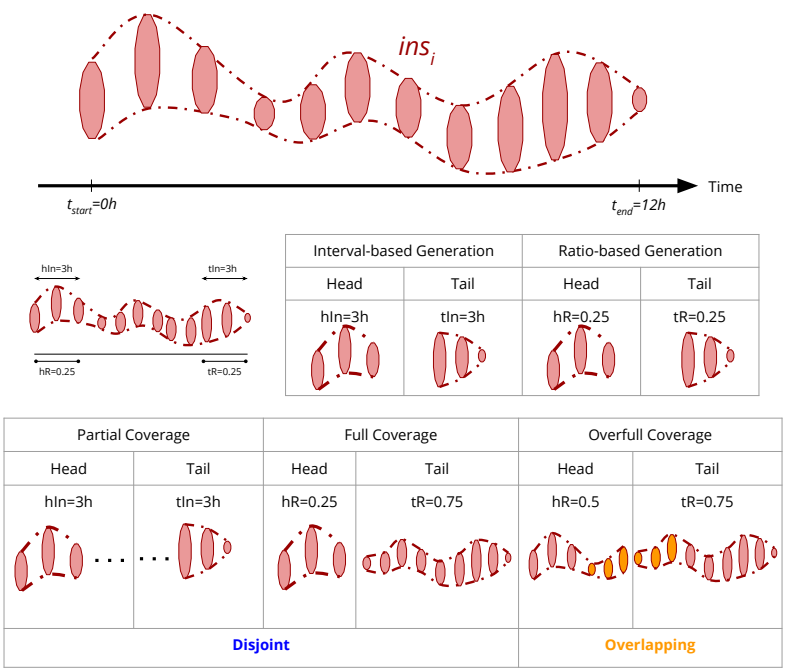

Figure 2: Head and tail generation strategies

In the ratio-based generation strategy, we are given two global parameters that are head ratio $(h R)$ and tail ratio $(t R)$. The ratios $(h R$ and $t R$ ) imply the proportion of trajectory's lifespan that will be assigned for head and tail segments, respectively. The lengths of the head and tail segments are variable, and dependent on the lifespan of the instances.

\subsubsection{Partial, Full and Overfull Coverage Strategies}

An important issue with the head and tail generation is the coverage of instance trajectories. In the full-coverage strategy the entire trajectory is divided into two parts, where the initial segment is considered as the head, and the last segment is considered as the tail. The full-coverage strategy is a constraint, where the entire instance trajectory is either a part of the head segment or a part of the tail segment. To actualize the full-coverage strategy, the ratio-based strategy is needed, where the sum of head and tail ratio must be 1 $(h R+t R=1)$. It can also be speculated that it is possible to use interval-based strategy for full-coverage; however, it requires the all instances in a dataset to have the same lifespan, which is far from reality.

In contrast to full-coverage, in the partial-coverage strategy, there can be portions of the instance trajectory, which are not covered by either of head or tail segments. Overfullcoverage occurs when the portions of the instance trajectory are covered by both head and tail segments. The partial and overfull coverage strategies are less constrained when compared to full coverage, and can be actualized by both interval and ratio-based strategies.

\subsubsection{Overlapping vs. Disjoint Strategies}

Another aspect of the head and tail generation is the characteristics of instance trajectory coverages. In disjoint strategy, no segment of the instance trajectory can be part of the both head and tail. Partial and full coverage strategies create disjoint head and tail segments. In the overlapping strategy, a portion of the instance trajectory can be included both in the head and tail segments. Overfull-coverage leads to the overlapping strategy.

In a particular dataset, overlapping (or disjoint) head and 
tail segments can be guaranteed by the ratio-based head and tail generation strategy. On the other hand, usage of interval-based generation can lead to a mixed coverage strategy, where head and tail segments can be overlapping or disjoint depending on the lifespan of the instance.

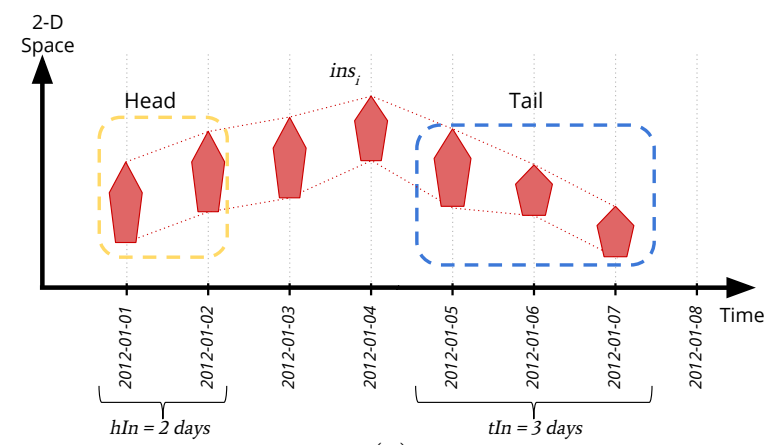

(a)

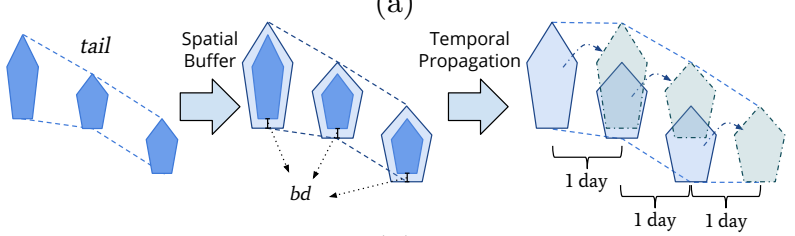

(b)

Figure 3: Creating the head and tail of an instance (a). Creating the tail window of an instance from the tail (b). (Parameters: $h$ In $=2$ days, $t$ In $=3$ days, and $t v=1$ day $)$

\subsubsection{Tail Window Generation}

The tail window is derived from the tail of the instance. The first operation for obtaining the tail window is the spatial buffer. Secondly, the spatially buffered geometries are propagated temporally. The amount of buffering is determined by buffer distance parameter (denoted as $b d$ ), while the period of temporal propagation is called tail validity period (denoted as $t v$ ). In other words, when creating the tail window, we initially enlarge the spatial region, then extend the spatial region for a specific period of time.

An example head and tail generation from an instance can be seen in Fig 3.a. In our example, we used intervalbased head and tail generation, and head interval is 2 days, and tail interval is 3 days. The initial 2-day segment of the instance, which corresponds to the first two recordings of the trajectory, is the head of the instance. Similarly, the final 3-day segment of the instance is the tail of the instance. An example of tail window generation can be seen in Fig 3.b. Firstly, the tail of the instance in Fig 3.a is spatially buffered. Then, each geometry in the buffered tail is considered to last its effect for another day $(t v=1 d a y)$, thus they are propagated in time for one day.

\subsection{Spatiotemporal Follow Relationship}

Given two instances $i n s_{i}$ and $i n s_{j}$, there exists a spatiotemporal follow relationship between ins $_{i}$ and $i n s_{j}\left(\right.$ ins $_{i}-$ $i n s_{j}$ ) if and only if (1) the start time of $i n s_{i}$ is less than the start time of $i n s_{j}$, and (2) there exists a spatiotemporal cooccurrence between the tail window of $i n s_{i}$ and the head of $i n s_{j}$. Under these conditions, $i n s_{i}$ is the followee and $i n s_{j}$ is the follower in the relationship.

To form a 2 -sequence, there must be one spatiotemporal follow relationship between two instances. More generally, to form a $k$-sequence, there must be $k$-1 spatiotemporal follow relationships between each consecutive participating instance. That is, for $k$ instances $\left(i n s_{1}, i n s_{2}, \ldots, i n s_{k}\right)$, the instance sequence $I S q=\left(i n s_{1}-i n s_{2} \longrightarrow \ldots\right.$ ins $\left.s_{k}\right)$ exists if and only if there exists a series of follow relationships between $i_{n} s_{1}$ and $i n s_{2}\left(i n s_{1}-i n s_{2}\right), i n s_{2}$ and $i n s_{3}\left(i n s_{2}-i n s_{3}\right), \ldots$, and, lastly $i n s_{k-1}$ and $i n s_{k}\left(i n s_{k-1}-i n s_{k}\right)$.

\subsection{Significance of the Instance Sequences}

An important aspect of the spatiotemporal event sequence mining is the determination of significant or spurious instance sequences. The significance assessment is important as the accuracy and reliability of resulting event sequences are dependent on the discovered instance sequences. For assessing the significance of the follow relationship between instances, we present the chain index.

The chain index, denoted as $c i$, for 2 -sequences are defined as the significance of spatiotemporal co-occurrence between tail window of the followee instance and head of the follower instance. The significance of spatiotemporal co-occurrences occurring between evolving region trajectories are studied in $[4,22]$. The significance can be measured with measures such as $O M A X, J$, or $J^{*}$. For this work, we will use the $J^{*}$ measure [4]. $J^{*}$ measure between two trajectory segments is defined as the ratio of intersection to union volume at time intervals where there exists a spatiotemporal overlap.

As previously mentioned, a $k$-sequence, where $k>2$, is essentially formed by $(k-1)$ follow relationships occurring between each consecutive instance pair. That is to say, there are $(k-1) 2$-sequences contained in a $k$-sequence. For sequences of length 3 or more, the chain index is defined as the minimum chain index of all 2-sequences contained.

Formally, for a 2-sequence, $I S q_{r}=\left(i n s_{r_{1}}-i n s_{r_{2}}\right)$, the significance of the follow relationship is assesed as follows:

$$
c i\left(I S q_{r}\right)= \begin{cases}J^{*}\left(t w_{r_{1}}, h_{r_{2}}\right) & \text { if } i n s_{r_{1}} \cdot t_{s}<i n s_{r_{2}} \cdot t_{s} \\ 0 & \text { otherwise }\end{cases}
$$

where $t_{s}$ represents the starting time of an instance, and $J^{*}$ for the tail window and head segments is defined as:

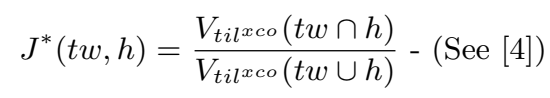

For a $k$-sequence $I S q_{i}=\left(\right.$ ins $\left._{i_{1}}-i n s_{i_{2}} \triangleright \ldots i n s_{i_{k}}\right)$, where $k>2$, the significance is assessed as follows:

$$
c i\left(I S q_{i}\right)=\min _{1 \leq j<k}\left(\operatorname{ci}\left(\text { ins }_{i_{j}}-i n s_{i_{j+1}}\right)\right)
$$

The instance sequences are considered as significant if their chain index value is greater than a user-defined chain index threshold $\left(c i_{t h}\right)$. The chain index is an antimonotonic measure. Antimonotonicity (downward closure property) is a crucial property for frequent pattern mining, as it helps pruning the search space efficiently. The property refers to the phenomenon that for any $k$-sequence, if the $k$-sequence is significant, any of its subsequences are also significant; and, the $k$-sequence cannot be significant, if at least one of its subsequences are not significant. Next, we will present the proof of antimonotonicity for the chain index.

Lemma: The chain index is antimonotonic.

Proof: Given $I S q_{j}=\left(i n s_{1}-i n s_{2} \triangleright \ldots i n s_{k}\right)$ is an instance sequence. Let $\operatorname{pre}_{j}$ be the length- $(k-1)$ prefix sub- 
sequence of $I S q_{j}$ and $s u f_{j}$ be the length- $(k-1)$ suffix subsequence of $I S q_{j}$.

$$
\begin{aligned}
& \operatorname{pre}_{j}=\left(i n s_{1}-i n s_{2} \triangleright \ldots>i n s_{k-1}\right) \\
& s u f_{j}=\left(i n s_{2}-i n s_{3} \triangleright \ldots>i n s_{k}\right) .
\end{aligned}
$$

For any chain index threshold $c i_{t h}$, if $I S q_{j}$ is significant:

$$
\begin{aligned}
& c i_{t h} \leq c i\left(I S q_{j}\right) \\
& c i_{t h} \leq \min \left(c i\left(i_{1}-i_{2}\right), \ldots, c i\left(i_{k-1}-i_{k}\right)\right) .
\end{aligned}
$$

The chain indexes of subsequences are defined as:

$$
\begin{aligned}
& c i\left(\text { pre }_{j}\right)=\min \left(c i\left(i_{1}-i_{2}\right), \ldots, c i\left(i_{k-2}-i_{k-1}\right)\right), \\
& c i\left(s u f_{j}\right)=\min \left(c i\left(i_{2}-i_{3}\right), \ldots, c i\left(i_{k-1}-i_{k}\right)\right),
\end{aligned}
$$

Then, $c i_{t h} \leq c i\left(I S q_{j}\right) \leq c i\left(\right.$ pre $\left._{j}\right)$ and $c i_{t h} \leq c i\left(\operatorname{sins}_{j}\right) \leq$ $c i\left(s u f_{j}\right)$, hence, chain index is antimonotonic.

\subsection{Prevalence of the Event Sequences}

Event sequences are derived from significant instance sequences. To measure how common a particular event sequence is, we will use participation index. The participation index is defined in [11], and signifies the importance of an event sequence. For an event sequence, $E S_{j}=\left(e_{j_{1}} \triangleright . . \triangleright e_{j_{k}}\right)$, the participation index of the event sequence is the minimum of participation ratios $(p r)$ of the event types in the sequence.

$$
p i\left(E S_{j}\right)=\min \left(\operatorname{pr}\left(e_{i_{1}} \mid E S_{j}\right), \ldots, p r\left(e_{i_{k}} \mid E S_{j}\right)\right)
$$

The participation ratio of an event type $\left(e_{i}\right)$ on an event sequence $\left(E S_{j}\right)$ is the ratio of number of unique participators of $e_{i}$ 's instances to the total number of event instances of $e_{i}$.

$$
\operatorname{pr}\left(\mathrm{e}_{i} \mid E S_{j}\right)=\frac{\mid\left\{\text { ins }_{i} \mid \text { ins }_{i} \in I S q_{i} \wedge i n s_{i} \cdot \mathcal{E}=e_{i} \wedge I S q_{i} \text { of-type } E S_{j}\right\} \mid}{\left|\mathbb{I}_{e_{i}}\right|}
$$

where $|\cdot|$ shows the set size. Event sequences are considered as prevalent, if and only if the participation index of the event sequence is greater than the user-defined participation index threshold $\left(p i_{t h}\right)$.

\section{DISCOVERY OF EVENT SEQUENCES}

In this section, we will discuss two algorithms that are $\mathrm{SE}-$ QuenceConnect and EsGrowth. SequenceConnect is an Apriori-based algorithm, where a bottom-up approach is applied. EsGrowth transforms the dataset into a directed graph structure (which is called event sequence graph), and discovers the spatiotemporal event sequences using a pattern growth-based approach.

\subsection{Initialization}

The initialization steps are shared in both of our algorithms. In these steps, we create the heads and tail windows of all the instances, and store them in the database. The pseudocode for the initialization steps can be seen in Alg. 1. For each instance, the initilization procedure creates head and tail windows as described in Section 3.1, and inserts them to the head $(H)$ and tail window $(T W)$ tables.

\subsection{SequenceConnect Algorithm}

The Apriori-based SequenceConnect algorithm is a bottom-up approach. It starts by discovering length-2 event sequences, and iteratively longer event sequences (of length$k$ ) are discovered from the event sequences (of length- $(k-1)$ )

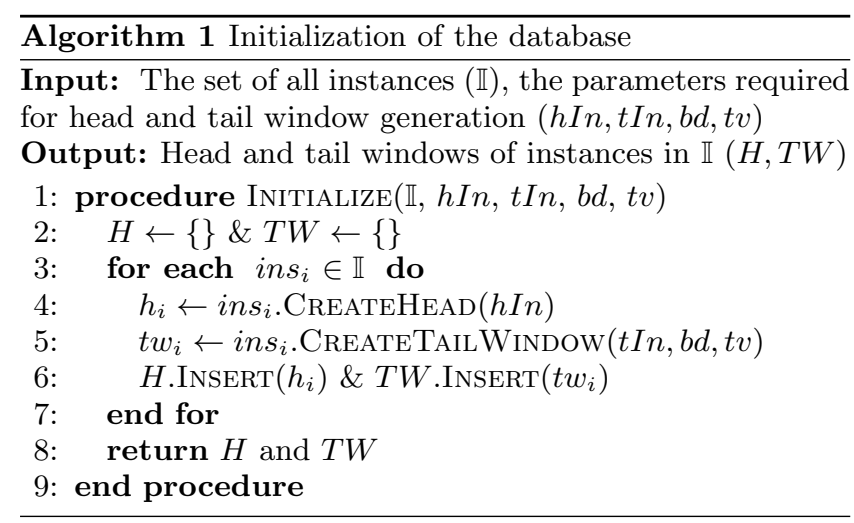

found in the previous step. At each step, the algorithm creates candidate event sequences, and tests whether those event sequences are prevalent by checking the prevalence of instance sequences as described in Section 3.4. Note that checking the prevalence requires determining the instance sequences of each event sequence. The event sequences are considered as prevalent if their participation index (calculated from instance sequences) is greater than the userdefined participation index threshold $\left(p i_{t h}\right)$.

The outline of the SEQUENCECONNECT algorithm is shown in Alg. 2. In steps 2 through 5, prevalent length-2 event sequences are discovered. Firstly, length-2 candidate event sequences $(c E S)$ are found by simply permuting the set of event types (with repetition). Then, for each of those candidates, significant 2-sequences are discovered (2S). In Step 5, the prevalence of the length-2 candidate event sequences are tested using the identified 2 -sequences. Non-prevalent candidates are filtered based on the prevalence index threshold.

Next, in the iterative steps 6 through 12 (of Alg. 2), we find longer size sequences by using the ones found in the previous iterations. At each iteration, firstly, candidate event sequences are generated. The candidate generation procedure (GEnERATECANDIDATES) is shown in the second part of Alg. 2. The procedure gets a pair of event sequences $\left(E S_{i}\right.$ and $E S_{j}$ ) from length- $k$ event sequence set. If the prefix of the first one $\left(E S_{i}\right)$ and suffix of the second one $\left(E S_{j}\right)$ is the same, then those two event sequences are merged into a length- $(k+1)$ event sequence, and added to candidate event sequence set $(c E S)$. Later, the instance sequences of the candidate event sequences are identified using the procedure called SEQUENCECONNECTOR, which can be seen in the third part of Alg. 2. This procedure iterates through the set of length- $k$ candidate event sequences, and for each candidate, it gets the length- $(k-1)$ prefix and suffix event sequences. The instance sequences of prefix and suffix event sequences are joined if they share $(k-2)$ instances. For instance, let a 3 -sequence (of-type prefix), IS $q_{1}$, be $\left(i n s_{1}-i n s_{2}-i n s_{3}\right)$. For $I S q_{1}$, the join operation essentially finds the instance sequences (of-type suffix) that starts with $i n s_{2}$ and $i n s_{3}$, and merges them with $I S q_{1}$. For the sake of example, let $I S q_{2}$ be $\left(i n s_{2}-i n s_{3}-i n s_{4}\right)$. The result of the join operation between $I S q_{1}$ and $I S q_{2}$ is a 4 -sequence $\left(i n s_{1}-i n s_{2}-i n s_{3}-i n s_{4}\right)$. This operation is continued for all the instance sequences.

After, the $k$-sequences are discovered, the prevalence of the candidate event sequences are tested. If they are prevalent, they are added to the prevalent event sequence set $E S$. If they are not prevalent, the instance sequences of them are 


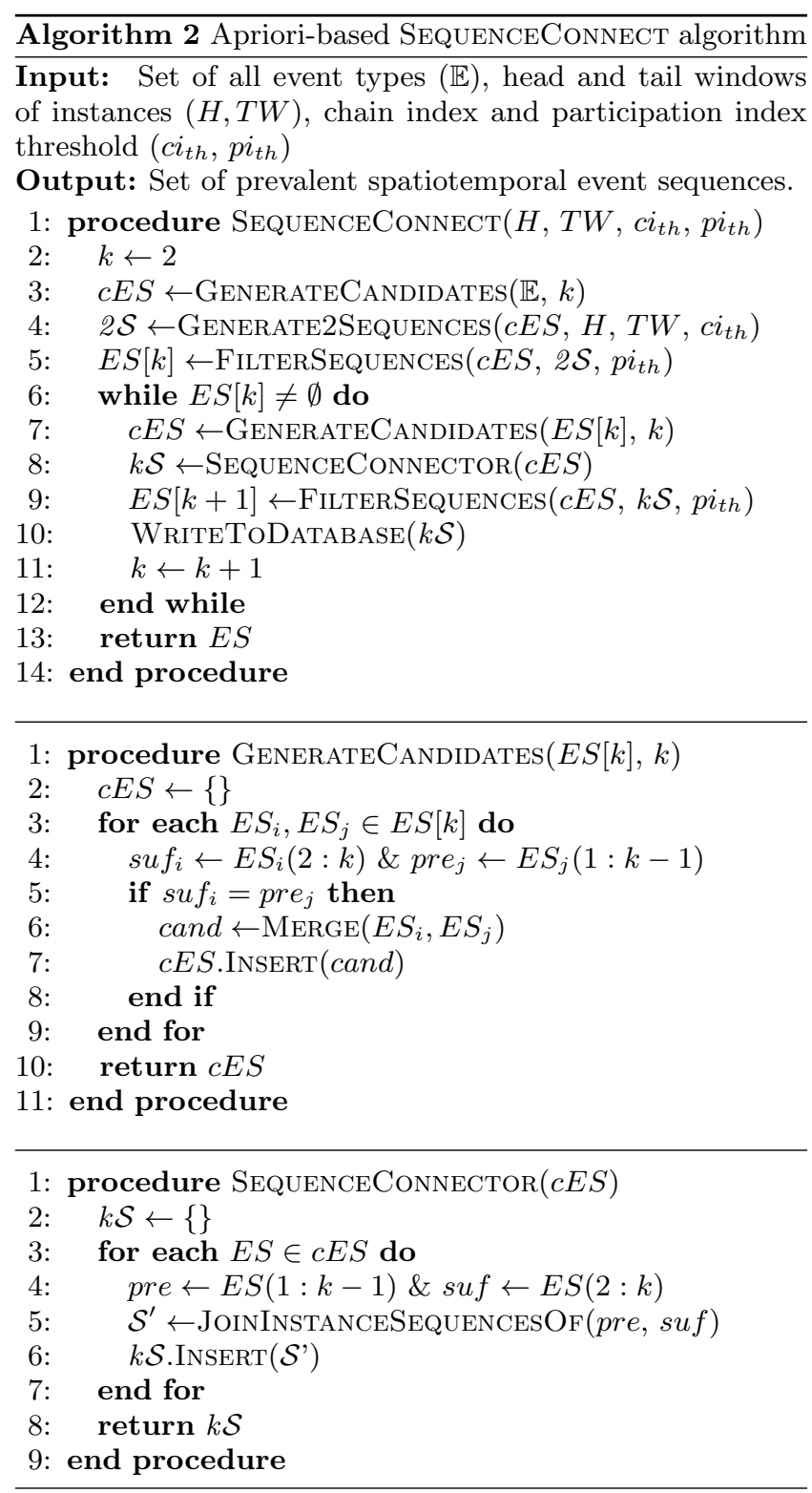

deleted from the set of significant $k$-sequences $(k S)$. Later, the $k$-sequences of prevalent event sequences are written to the database to be used in further iterations. The iterative steps are terminated when no prevalent event sequences are discovered in a step.

\subsection{EsGrowth Algorithm}

Our second STES mining algorithm is EsGROwTH, which stands for Event Sequence Growth. EsGrowth initially discovers the significant follow relationships appearing between the instances. These relationships are transformed into a directed acyclic graph structure. Using the graph structure, the algorithm recursively discovers the frequently appearing event sequences using a pattern growth-based approach. The outline of EsGrowTH can be seen in Alg. 3.

Similar to the SEquenceConnect algorithm, the EsGROWTH algorithm initially discovers the 2 -sequences ( $2 \mathcal{S}$ ) to identify the significant follow relationships. In step 4 of Alg. 3, the 2-sequences $\left(i n s_{i}-i n s_{j} \in 2 \mathcal{S}\right)$ are transformed

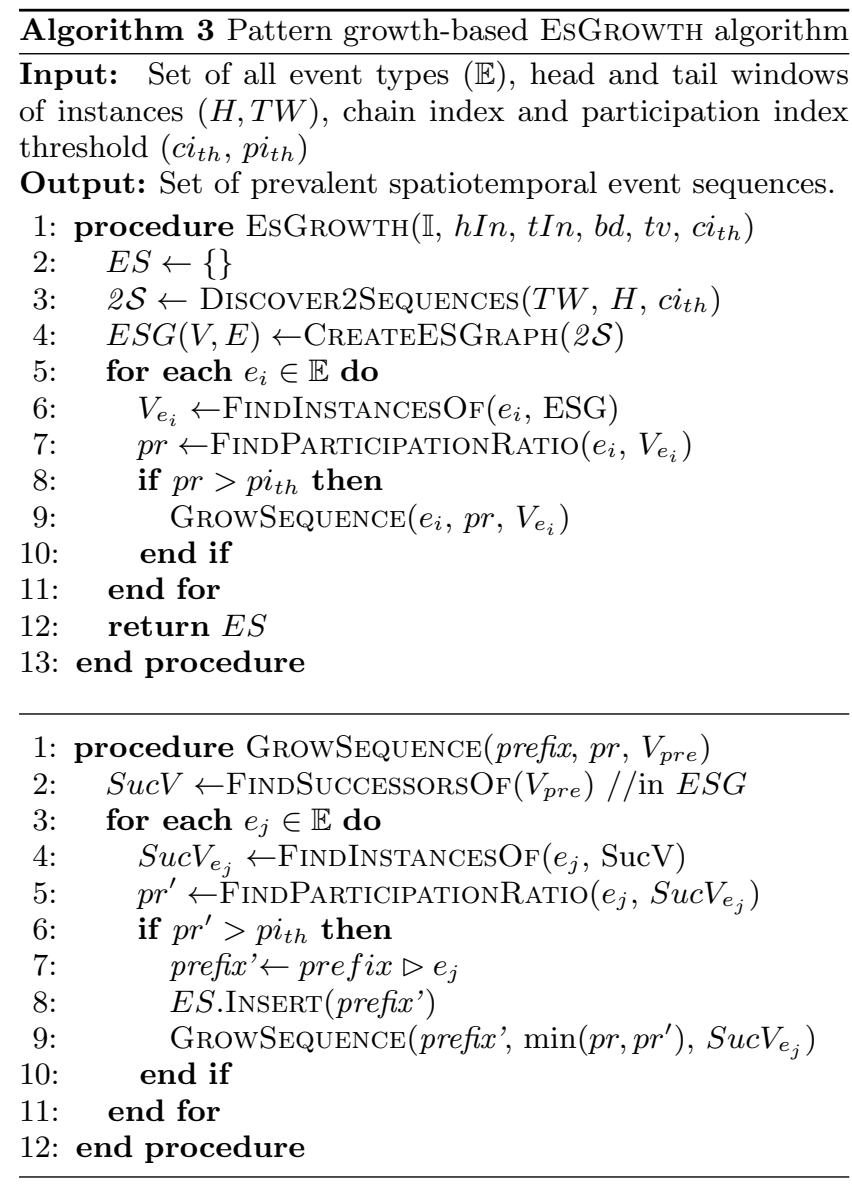

into the event sequence graph structure (denoted as $E S G$ ). The transformation is as follows: For each 2-sequence, in the form of $i n s_{i}-i n s_{j}$, two vertices corresponding to the instances, and a directed edge between those two vertices are added to the graph. The vertices only store the unique identifier and event type of the instance. The edges are directed from followee to follower. For a 2-sequence, $i n s_{i}-i n s_{j}$, we create two vertices $\left(' i: i n s_{i} \cdot \mathcal{E} '\right.$ and ' $j: i n s_{j} . \mathcal{E}$ '), and add them to vertex set of the graph if they are not already inserted. Then, a directed edge from the vertex ' $i: i n s_{i} \mathcal{E}^{\prime}$ to ' $j: i n s_{j} . \mathcal{E}$ ' is added to the edge set of $E S G$.

Next, the algorithm recursively discovers the event sequences using the event sequence graph. This part of our algorithm extends the pattern growth-based PrefixSpan algorithm [20] to the event sequence graphs. Initially, the algorithm starts by iterating the event types $\left(e_{i}\right)$ in the set of all events $(\mathbb{E})$. For each event type, we identify the nonleaf vertices, which corresponds to the instances of the event type (Step 6). Then a participation ratio $(p r)$ is calculated from those vertices to check the prevalence using $p i_{t h}$. If $p r$ is greater than the $p i_{t h}$, we apply the recursive GrowSEQUENCE procedure (Step 8 through 10).

The GrowSEquence procedure is shown in the second part of Alg. 3. The procedure takes a prefix event sequence ( $p r e f i x)$, the current minimum participation ratio value $(p r)$ for the prefix sequence, and a set of pointers to the vertices $\left(V_{\text {pre }}\right)$ as parameters. In essence, the participation ratio parameter $(p r)$ is the participation index value for the prefix event sequence; nevertheless, we choose to keep it as $p r$ 
because the procedure is also called with length-1 prefixes, which are not event sequences by definition. The vertices in $V_{\text {pre }}$ correspond to the last discovered vertices in the paths, which virtually forms the instance sequences of-type prefix in the event sequence graph. The procedure proceeds as follows. Firstly, the successors (immediate neighbors) of the vertices in $V_{\text {pre }}$ are found and added to the successor vertex set $(S u c V)$. Then, for each event type $e_{j}$, a subset of successor vertex set (containing the instances of $e_{j}$ ) is created (Step 4 of GrowSEQuence procedure in Alg. 3 - denoted as $\left.S u c V_{e_{j}}\right)$. For readers who are familiar with the PrefixSpan algorithm, the set of successer vertices, $S u c V_{e_{j}}$, has a similar functionality with the prefix-projected databases [20]. In contrast to prefix-projected databases, we only pass pointers to the vertices of the graph, which significantly reduces the storage requirements of the algorithm. After identifying successors, a temporary participation ratio value $\left(p r^{\prime}\right)$ is calculated for $e_{j}$. If $p r^{\prime}$ is greater than the participation index threshold, we extend the prefix event sequence with $e_{j}$, and create a new prefix event sequence prefix' (Step 7 of GrowSequence procedure). Note that, we already know that the participation ratio for prefix, that is $p r$ (given as parameter), is certainly greater than $p i_{t h}$ as it is checked before; therefore, only the temporary participation ratio value $\left(p r^{\prime}\right)$ is checked. At this point, prefix' is guaranteed to be prevalent, thus is inserted to the set of prevalent event sequences, ES. Lastly, the GrowSEQUENCE procedure is called with the newly created prefix event sequence, prefix'. Along with the prefix', the minimum of old and new participation ratio $\left(\min \left(p r, p r^{\prime}\right)\right)$, and the vertex subset formed by the successors $\left(S u c V_{e_{j}}\right)$ are passed as parameters. Note that the base case in our recursion occurs when the temporary participation ratio is less than the participation index threshold. In this case, the prefix', which is created by appending the new event type to prefix, is not prevalent. Therefore, there is no need to check the longer length event sequences generated from prefix'.

\section{EXPERIMENTAL EVALUATION}

In this section, we evaluate the performance of spatiotemporal event sequence mining algorithms. Our algorithms are implemented in Java programming language, datasets are stored in PostgreSQL database, and the experiments were conducted on a personal computer with Intel Core i7 3.4 $\mathrm{GHz} \mathrm{CPU}$ and 8GB memory.

\subsection{Datasets and Experimental Settings}

To analyze the performance of our algorithms, we used three real-life solar event datasets. These datasets include spatiotemporal instances of six different solar event types that are: Active Regions (AR), Coronal Holes (CH), Emerging Flux (EF), Filaments (FI), Sigmoids (SG), and Sunspots (SS). The event instances are obtained from Heliophysics Event Knowledgebase [1], and consist of individual spatial boundaries of the solar events at a particular time (i.e., timegeometry pairs).

The individual recordings are tracked with the algorithm presented in [13], and interpolated at every 10-minute time interval. The datasets are named as $1 \mathrm{Mo}, 3 \mathrm{Mo}$, and $6 \mathrm{Mo}$. $1 \mathrm{Mo}$ dataset covers the solar event instances from January 2013. 3Mo dataset covers the solar event instances from February 1, 2013 to April 30, 2013. 6Mo dataset covers the solar event instances from May 1, 2013 to November
Table 1: Characteristics of Datasets

\begin{tabular}{ccccc}
$\begin{array}{c}\text { Dataset } \\
\text { Name }\end{array}$ & $\begin{array}{c}\text { Number of } \\
\text { Instances }\end{array}$ & $\begin{array}{c}\text { Number of Time } \\
\text { Geometry Pairs }\end{array}$ & $\begin{array}{c}\text { Average } \\
\text { Lifespan }\end{array}$ & $\begin{array}{c}\text { Interpolation } \\
\text { Interval }\end{array}$ \\
\hline 1Mo & 1,152 & 193,730 & 28.03 hours & 10 minutes \\
3Mo & 3,106 & 480,136 & 25.76 hours & 10 minutes \\
6Mo & 6,866 & 922,323 & 22.39 hours & 10 minutes
\end{tabular}

30, 2013. The characteristics of our datasets such as number of instances, time-geometry pairs, average lifespans are displayed in Table 1.

The experiments were performed on the above-mentioned datasets with varying head and tail intervals, buffer distance, and tail validity interval values. The thresholds were kept constant throughout the experiments. Participation index threshold is set to $10^{-5}$, and chain index threshold is set to $10^{-3}$. For each set of experimental runs, buffer distance, head interval and tail interval values are set to same values. Tail interval values are varied and set to $0,1,2,3$, and 4 hours for each set. We show the parameters of eash set of runs in Table 2. Head and tail interval values are set to $1,2,3$, and 4 hours. When head and tail intervals are set to 1 or 2 hours, we set the buffer distance to a smaller 25 arcsec, while for 3 and 4 hours, we set the buffer distance to 50 arcsec. Each set is run 5 times with varying tail validity values. Each of those experiments were applied all three of our datasets for both of our algorithms. Meaning we conducted 120 experimental runs in total.

Table 2: Parameter Settings of Experimental Runs

\begin{tabular}{ccccc}
$\begin{array}{c}\text { Experiment } \\
\text { Set Name }\end{array}$ & $\begin{array}{c}\text { Head } \\
\text { Interval }\end{array}$ & $\begin{array}{c}\text { Tail Window } \\
\text { Interval }\end{array}$ & $\begin{array}{c}\text { Tail Validity } \\
\text { Interval }\end{array}$ & $\begin{array}{c}\text { Buffer } \\
\text { Distance }\end{array}$ \\
\hline H-TW-1h & 1 hour & 1 hour & $\{0,1,2,3,4\}$ hours & 25 arcsec \\
H-TW-2h & 2 hour & 2 hour & $\{0,1,2,3,4\}$ hours & 25 arcsec \\
H-TW-3h & 3 hour & 3 hour & $\{0,1,2,3,4\}$ hours & 50 arcsec \\
H-TW-4h & 4 hour & 4 hour & $\{0,1,2,3,4\}$ hours 50 arcsec
\end{tabular}

\subsection{Initialization and 2-sequence Generation}

In this part we will discuss two major steps shared by both of our algorithms, that are head and tail window generation (initialization) and the generation of significant 2-sequences. We use the same code for those two steps; therefore, we will report the average of the running times of two algorithms.

Fig. 4 demonstrates the running time required for initialization steps when head and tail interval values are set to 4 hours (with different tail validity values). As expected, the running time for these steps increases with respect to the number of instances and the tail validity values. In Fig. 4.b, we show the average time (per instance) required for creating head and tail window of an instance. It can be seen that on average the generation values are very similar for each different tail validity value.

The 2-sequence generation is essentially a series of spatiotemporal join operations with the spatiotemporal overlap predicate between tail windows of the followees and heads of the followers. As we have six different event types in all of our datasets, we perform 36 joins. We used a temporal $\mathrm{B}^{+}$-tree index on all of our head and tail window tables in the database to increase the performance of these joins. The join operations ultimately find all overlapping head and tail window pairs for all possible length- 2 event sequences, and later calculates the $J^{*}$ value of the follow relationships, 


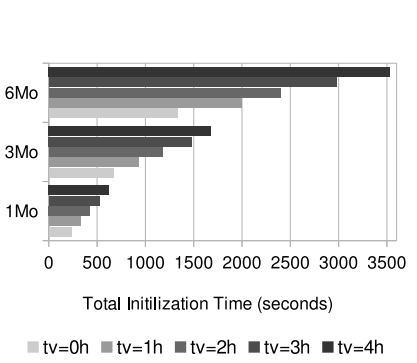

(a)

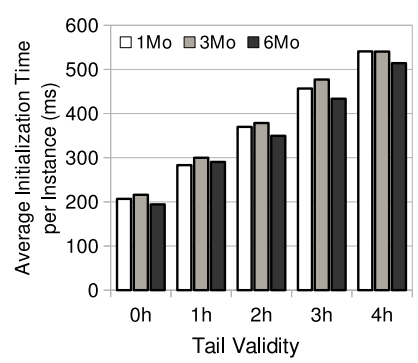

(b)
Figure 4: Time required for creating the head and tail windows (initilization) when head and tail interval are set to 4 hours (See Table 2 - H-TW-4h). (a) Total initialization with different tail validity values. (b) Average time required per instance in the initialization phase.

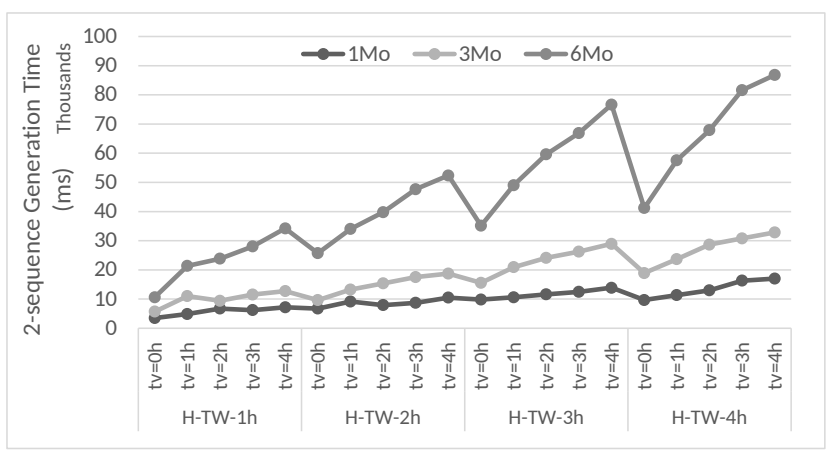

Figure 5: Running times for generation of 2-sequences

which is used for significance analysis.

In Fig. 5, we show the running times required for generating significant 2-sequences for all experimental settings and for all datasets. Firstly, we can observe that increasing the head and tail interval values moderately increase the 2sequence generation time. This is much expected, because the number of polygons in head and tail windows increases with respect to head and tail interval values; and, this leads to greater number spatiotemporal overlaps, as well as the $J^{*}$ calculations taking longer times. More importantly, we observe an increase in the running times with respect to the tail validity values. Especially for $6 \mathrm{Mo}$ dataset this situation is more visible, where time requirements increase more than two times for generating 2 -sequences when tail validity is increased from 0 hours to 4 hours.

\subsection{STES Discovery}

In this part of our experiments, we will analyze the total running time requirements of our algorithms apart from the shared initialization and 2-sequence generation procedures, which we discussed earlier. We demonstrate the total running times required for discovering the event sequences in Fig. 6. For the purpose of better visibility, the results are grouped for each dataset $(1 \mathrm{Mo}, 3 \mathrm{Mo}$, and $6 \mathrm{Mo})$ in subfigures Fig. 6.a, Fig. 6.b, and Fig. 6.c.

In a nutshell, we can observe that EsGROWTH algorithm is generally more efficient than SEQUENCECONNECT algorithm. The first thing that attracts attention is the effect of parameters. We can see that longer head and tail intervals increase the running time with a few exceptions. Similarly, longer tail interval values generally increase the running times for both algorithms. This is an anticipated result, because for both algorithms the rise in tail validity, head interval, or tail interval values proliferates the number of instance sequences, which leads to greater running times for algorithms.

More interesting result is the quick spikes in running times of the algorithms when tail validity is increased from 0 to 1 hour. This essentially shows that we capture more 2sequences (i.e. follow relationships). Capturing more follow relationships results in creation of longer instance sequences, and eventually longer event sequences. Therefore, the event sequence discovery requires longer running times. To complement these results, we show the total number of vertices and edges inserted the event sequence graphs in Fig. 7. As mentioned earlier, the edges represent the number of significant follow relationships or 2 -sequences. Note that 0 hours tail validity value means that we do not propagate the tails of the instances in time dimension, meaning the tails are only spatially buffered but temporally not extended.

From Fig. 7, we can also recognize that increasing the tail validity grows the number of instance sequences faster increasing the head and tail intervals. This is a significant result, because while tail window generation is a computationally expensive procedure, it is effective in the discovery of sequence forming behavior, given the spikes in the running times of the algorithms. For instance, in the experiments with $6 \mathrm{Mo}$ dataset, increasing the head and tail interval from 1 hour $(\mathrm{H}-\mathrm{TW}-1 \mathrm{~h}, \mathrm{tv}=0 \mathrm{~h})$ to 4 hours $(\mathrm{H}-\mathrm{TW}-4 \mathrm{~h}, \mathrm{tv}=0 \mathrm{~h})$ results in three times increase in the number of significant follow relationsips (from approximately 500 to 1500). On the other hand, increasing the tail validity from 0 to 4 hours (in H-TW-1h) increases the number of significant follow relationships roughly 5 times (from approximately 500 to 2500).

Another observation we can make is the increase in the running times for SEQUENCECONNECT is much faster than the EsGrowth. Apart from the spikes caused by 0 to 1 hour tail validity increase, the running time for EsGROwTH follows a much smoother increase, while the increase in $\mathrm{SE}$ QUENCECONNECT appears to be very steep. There can be multiple reasons behind this behavior. Firstly, EsGrowTH operates on a directed acyclic graph structure (i.e. event sequence graph), which is much more efficient for searching the followers of an instance. In addition to that, it avoids the candidate generation. In contrast, SEQUENCEConnect algorithm performs computationally expensive join operations, which reduces the efficiency when processing larger number of instances and follow relationships. This situation is more visible in the experiments in 6Mo datasets. We see larger differences between the running times as tail validity values are increased. Another reason for this behavior may be related to the lower participation index thresholds. Apriori-based algorithms are effective in reducing the search space using the user-defined thresholds. Nevertheless, a low participation index value simply discovers every possible event sequence, resulting in a decrease of the SEQUENCEConneCT algorithm's pruning power. For EsGrowTH algorithm, the event sequence generation is an overhead; however, the recursive GROWSEQUENCE procedure is not much affected from higher number of follow relationships as the graph structure allows efficient in-memory search for the discovery of potential instance sequences.

Overall, we can conclude that EsGrowTH algorithm is more efficient than SEQUENCECONNECT algorithm, because 


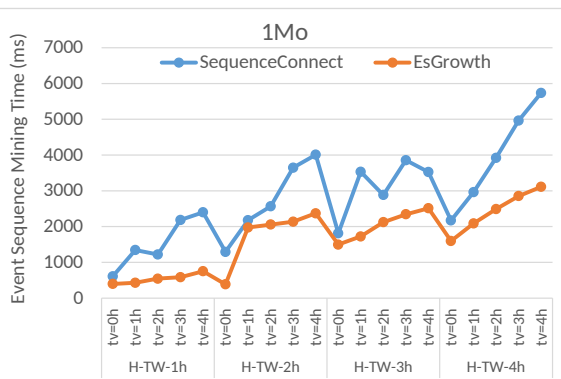

(a)

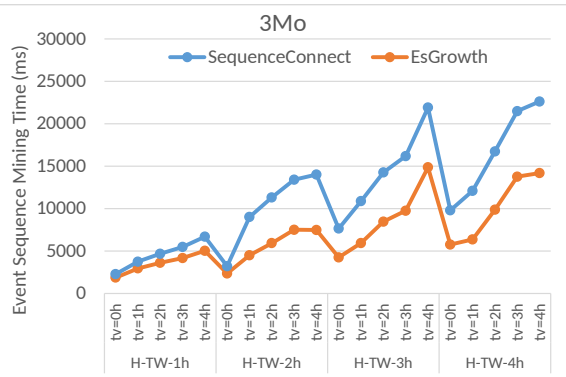

(b)

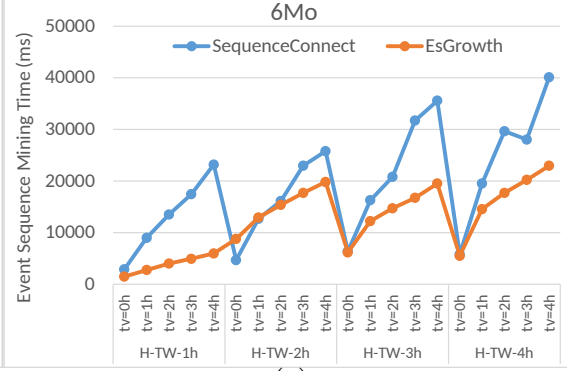

(c)

Figure 6: The total running time required for SEQuenceConnect and EsGrowth algorithms under different head interval, tail interval, and tail validity settings. (a), (b), and (c) shows the results for $1 \mathrm{Mo}$, $3 \mathrm{Mo}$, and $6 \mathrm{Mo}$ datasets respectively.

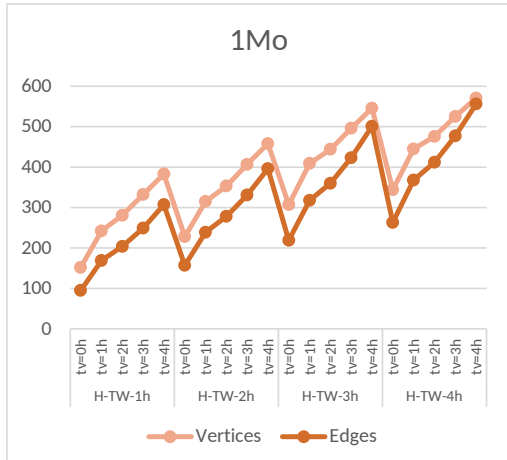

(a)

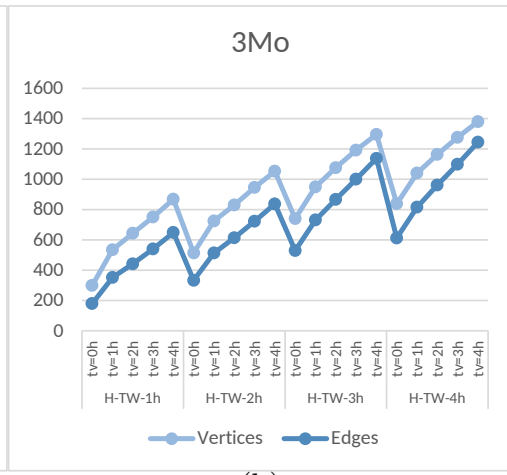

(b)

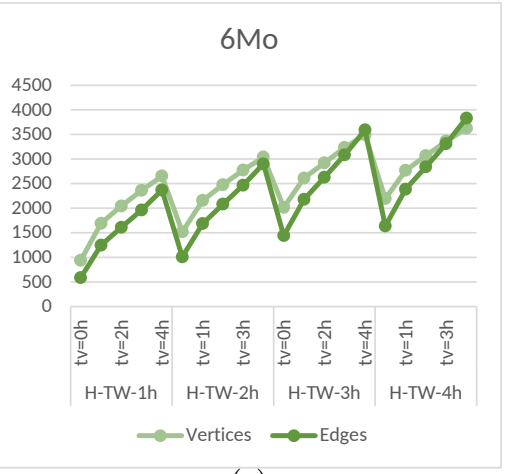

(c)

Figure 7: The characteristics of the created event sequence graphs under different parameter settings grouped by the datasets. The number of edges corresponds to the number of discovered 2-sequences.

it avoids the expensive candidate generation and filtering steps. It is also worth noting that, SEQuenceConNECT writes the significant instance sequences back to the database, which is an extra overhead. EsGrowth, on the other hand, exploits the efficient searching capabilities of event sequence graph structure.

\section{CONCLUSION AND FUTURE WORK}

In this work, we have introduced spatiotemporal event sequences and presented two mining algorithms: SEQUENCEConnect and EsGrowth. The SequenceConnect algorithm is Apriori-based and follows a candidate generation and testing process. The EsGrowTH algorithm is a pattern growth-based algorithm. It generates an event sequence graph, which is a directed acyclic graph structure, and grows the instance sequences by recursively searching the graph structure.

In addition to the algorithms, we defined a flexible spatiotemporal follow relationship, which captures the sequence forming behavior between the spatiotemporal instances. We proposed an antimonotonic significance measure, chain index, for assessing the importance of instance sequences. Both of our algorithms use participation index for determining the prevalence of the event sequences. Both measures carry the downward closure property, which enables our algorithms to more efficiently discover the event sequences.

We performed an extensive experimental evaluation for comparing the efficiency of our algorithms using different parameters and datasets. Based on the results of our ex- periments, we can conclude that pattern growth-based EsGrowth algorithm performs better than SEQuenceConNECT algorithm.

Our future work lies in dynamic parameter assessments for head and tail window generation with statistical evaluation. Furthermore, we also aim to study the substructures in the generated event sequence graphs, as well as the statistical significance of the generated event sequences.

\section{ACKNOWLEDGMENTS}

This work was supported in part by two NASA Grant Awards (No. NNX11AM13A, and No. NNX15AF39G), and one NSF Grant Award (No. AC1443061). The NSF Grant Award has been supported by funding from the Division of Advanced Cyberinfrastructure within the Directorate for Computer and Information Science and Engineering, the Division of Astronomical Sciences within the Directorate for Mathematical and Physical Sciences, and the Division of Atmospheric and Geospace Sciences within the Directorate for Geosciences.

\section{REFERENCES}

[1] Heliophysics Event Knowledgebase, May 2016. [accessed 10-May-2016].

[2] R. Agrawal and R. Srikant. Mining sequential patterns. In Proc. of the Eleventh Int. Conf. on Data Engineering, March 6-10, 1995, Taipei, Taiwan, pages 3-14, 1995. 
[3] J. F. Allen. Maintaining knowledge about temporal intervals. Commun. ACM, 26(11):832-843, 1983.

[4] B. Aydin, V. Akkineni, and R. Angryk. Time-efficient significance measure for discovering spatiotemporal co-occurrences from data with unbalanced characteristics. In Proc. of the 23rd ACM SIGSPATIAL Int. Conf. on Advances in Geographic Information Systems, pages 80:1-80:4. ACM, 2015.

[5] B. Aydin and R. A. Angryk. A graph-based approach to spatiotemporal event sequence mining. In IEEE Int. Conf. on Data Mining Workshop, ICDMW 2016, Barcelona, Spain, December 12-15, 2016, 2016.

[6] B. Aydin and R. A. Angryk. Spatiotemporal event sequence mining from evolving regions. In 23rd Int. Conf. on Pattern Recognition, ICPR 2016, Cancun, Mexico, December 4-8, 2016, 2016.

[7] H. Cao, N. Mamoulis, and D. W. Cheung. Mining frequent spatio-temporal sequential patterns. In Proceedings of the 5th IEEE International Conference on Data Mining (ICDM 2005), 27-30 November 2005, Houston, Texas, USA, pages 82-89, 2005.

[8] G. Dong and J. Pei. Sequence Data Mining, volume 33 of Advances in Database Systems. Kluwer, 2007.

[9] M. Erwig. Toward spatio-temporal patterns. In R. de Caluwe, G. de TrÃl', and G. Bordogna, editors, Spatio-Temporal Databases, pages 29-53. Springer Berlin Heidelberg, 2004.

[10] F. Giannotti, M. Nanni, F. Pinelli, and D. Pedreschi. Trajectory pattern mining. In Proceedings of the 13th $A C M$ SIGKDD International Conference on Knowledge Discovery and Data Mining, San Jose, California, USA, August 12-15, 2007, pages 330-339, 2007.

[11] Y. Huang, S. Shekhar, and H. Xiong. Discovering colocation patterns from spatial data sets: A general approach. IEEE Trans. Knowl. Data Eng., 16(12):1472-1485, 2004.

[12] Y. Huang, L. Zhang, and P. Zhang. A framework for mining sequential patterns from spatio-temporal event data sets. IEEE Trans. Knowl. Data Eng., 20(4):433-448, 2008.

[13] D. Kempton, K. G. Pillai, and R. A. Angryk. Iterative refinement of multiple targets tracking of solar events. In 2014 IEEE Int. Conf. on Big Data, Big Data 2014, Washington, DC, USA, October 27-30, 2014, pages 36-44, 2014.

[14] S. R. Langhoff and T. Straume. Highlights of the "Space Weather Risks and Society" workshop. Space Weather, 10(6), 2012.

[15] C. Mooney and J. F. Roddick. Sequential pattern mining - approaches and algorithms. ACM Comput. Surv., 45(2):19, 2013.

[16] F. Mörchen. Tutorial CIDM-T temporal pattern mining in symbolic time point and time interval data. In Proc. of the IEEE Symposium on Computational Intelligence and Data Mining, Nashville, TN, USA, March 30-April 2, 2009, 2009.

[17] R. Moskovitch and Y. Shahar. Fast time intervals mining using the transitivity of temporal relations. Knowl. Inf. Syst., 42(1):21-48, 2015.

[18] P. Papapetrou, G. Kollios, S. Sclaroff, and
D. Gunopulos. Discovering frequent arrangements of temporal intervals. In Proc. of the 5th IEEE Int. Conf. on Data Mining (ICDM 2005), 27-30 November 2005, Houston, Texas, USA, pages 354-361, 2005.

[19] J. Pei, J. Han, B. Mortazavi-Asl, H. Pinto, Q. Chen, U. Dayal, and M. Hsu. Prefixspan: Mining sequential patterns by prefix-projected growth. In Proc. of the 17th Int. Conf. on Data Engineering, April 2-6, 2001, Heidelberg, Germany, pages 215-224, 2001.

[20] J. Pei, J. Han, B. Mortazavi-Asl, J. Wang, H. Pinto, Q. Chen, U. Dayal, and M. Hsu. Mining sequential patterns by pattern-growth: The prefixspan approach. IEEE Trans. Knowl. Data Eng., 16(11):1424-1440, 2004.

[21] J. Pei, J. Han, B. Mortazavi-Asl, and H. Zhu. Mining access patterns efficiently from web logs. In Knowledge Discovery and Data Mining, Current Issues and New Applications, 4th Pacific-Asia Conference, PADKK 2000, Kyoto, Japan, April 18-20, 2000, Proceedings, pages 396-407, 2000.

[22] K. G. Pillai, R. A. Angryk, and B. Aydin. A filter-and-refine approach to mine spatiotemporal co-occurrences. In 21st SIGSPATIAL International Conference on Advances in Geographic Information Systems, SIGSPATIAL 2013, Orlando, FL, USA, November 5-8, 2013, pages 104-113, 2013.

[23] H. A. Salas, S. Bringay, F. Flouvat, N. Selmaoui-Folcher, and M. Teisseire. The pattern next door: Towards spatio-sequential pattern discovery. In Advances in Knowledge Discovery and Data Mining - 16th Pacific-Asia Conf., PAKDD 2012, Kuala Lumpur, Malaysia, May 29 - June 1, 2012, Proc., Part II, pages 157-168, 2012.

[24] R. Srikant and R. Agrawal. Mining sequential patterns: Generalizations and performance improvements. In Advances in Database Technology EDBT'96, 5th International Conference on Extending Database Technology, Avignon, France, March 25-29, 1996, Proceedings, pages 3-17, 1996.

[25] F. Verhein. Mining complex spatio-temporal sequence patterns. In Proceedings of the SIAM International Conference on Data Mining, SDM 2009, April 30 May 2, 2009, Sparks, Nevada, USA, pages 605-616, 2009.

[26] E. Winarko and J. F. Roddick. ARMADA - an algorithm for discovering richer relative temporal association rules from interval-based data. Data Knowl. Eng., 63(1):76-90, 2007.

[27] M. J. Zaki. SPADE: an efficient algorithm for mining frequent sequences. Machine Learning, 42(1/2):31-60, 2001.

[28] C. Zhang, J. Han, L. Shou, J. Lu, and T. F. L. Porta. Splitter: Mining fine-grained sequential patterns in semantic trajectories. PVLDB, 7(9):769-780, 2014.

[29] Y. Zhao, H. Zhang, L. Cao, H. Bohlscheid, Y. Ou, and C. Zhang. Data mining applications in social security. In Data Mining for Business Applications, pages 81-96. Springer, 2009. 